\section{Improving the evaluation of milestones for students completing a clinical genetics elective}

To the Editor: We read the article by Press and Bodurtha, "Milestones for Medical Students Completing a Clinical Genetics Elective,"1 which described how a milestone template for evaluating and improving the genetics elective was successfully introduced for third- and fourth-year students at the Johns Hopkins School of Medicine (Baltimore, Maryland), with particular interest. The work was motivated by previous initiatives by the Accreditation Council for Graduate Medical Education, which used templates, regarding residents and fellows, contributing to the development of milestones and evaluation-driven methods. The milestone template, which included an evaluation guide (5 levels) and 49 competencies, was created via feedback from eight medical students and eight assistants.

We believe that the selection of competencies was extensive and coherently distributed in groups (patient care, medical knowledge, professionalism, practice-based performance improvement, interpersonal and communication skills, systems-based practice) that are necessary for constructing skills for medical genetics practice. We have determined that it adds useful information (competencies) for our own evaluation methods. However, the template might be enriched by some extra milestones and, when used by other academic structures, be modified depending on specific particularities.

We are a group of professors (medical geneticists and scientists working on molecular medicine) who, for 30 years, have taught basic sciences and medical genetics to medical students at a private university (Universidad del Rosario) in Colombia. Colombia is a lower-middle-income country that has evolved considerably over the past 10 years, reflecting numerous encouraging changes in academic and research structures in other South American countries. The curriculum for medicine in our institution has evolved from a system focused on the teaching of subjects (e.g., basic and medical genetics, electives) to an integrated learning plan, based on acquiring specific competencies. ${ }^{2}$ The curriculum, which encompasses basic biomedical sciences, clinical teaching, sociohumanistic knowledge (including medical professionalism), and population health, is based on a teaching and learning system (the integrated learning activities-based system), which enhances the development of competencies by following nonmemory and analytic methodologies. Medicine (and genetics) teaching is therefore constructed around expected learning results that integrate knowledge from global and specific perspectives. Course structure is flexible, allowing elective/selective components to be chosen. Students can choose to follow a coterminal path toward MSc/ $\mathrm{PhD}$ degrees allowing scientific research or take on a second role focused on clinical features (e.g., fellow or resident). Our students can opt into genetics courses throughout their study program and can follow a clinical genetics elective.

Concerning the competencies included by Press and Bodurtha $^{1}$ in their milestone template, we have observed that most of them are successfully evaluated by teachers and acquired by our students. ${ }^{1}$ However, some of them are integrated only partially or not at all (Supplementary Table S1 online).

The competency "understands basic diagnosis and management of metabolic genetic conditions" is not acquired by our students due to the lack of a well-established national system for diagnosing and treating patients suffering from these diseases. Thus, because of fluctuating access to such patients, students have limited practical skills regarding these pathologies. The situation is similar to that observed in other countries in the region (e.g., Bolivia, Peru, Panama, and Ecuador) where these kinds of diseases are not treated in specialized medical centers. However, our students have the possibility to review, especially from a bioethics standpoint, the national laws regulating orphan diseases.

We have also observed that our undergraduate students lack the appropriate competencies defined by Press and Bodurtha ${ }^{1}$ as "distinguishes different types of genetic tests and suggests appropriate genetic testing" and "recognizes the differences between genetic and genomic analysis." Although genetic testing is available in Colombia, patient access is restricted because such tests are particularly expensive; furthermore, these services are sold by nonacademic private companies, thereby limiting students' chances of coming into contact with them, especially with new genetic tests. It is worth noting that students from our genetics and genomics research group, the Center For Research in Genetics and Genomics, particularly those following MSc and $\mathrm{PhD}$ courses, have the opportunity to work on robust genetic and genomics techniques. Indeed, our work on genomics research has been considered one of the few successful initiatives on this topic in the region although we consider Press and Bodurtha's template necessary to support instruction in these competencies to our undergraduate medical students. ${ }^{3}$

The competency "understands the purpose and procedure of newborn screening" is partially acquired by our students. In fact, although they can appreciate the importance of genetic newborn screening, they focus on a unique test (thyroid hormone screening) that has been made mandatory by Colombian law. Two other competencies are only partially learned by our students: "counsels patient about how genetic 
testing works and its benefits and limitations" and "understands more complex genetics." These topics are not covered in depth in terms of theoretical and practical knowledge; thus, instruction in these areas needs improvement.

However, we have identified three competencies in our medical students that were not recorded by Press and Bodurtha $^{1}$ (Supplementary Table S1). First, owing to the structure of our curriculum and system of electives, which enables students to learn genetics throughout their studies, we have detected competencies regarding concepts of translational medicine. The students are capable of interpreting diseases (rare and common) in the light of molecular genetics to establish potential applications in clinics. Second, the Colombian population is formed by the mixing of diverse ethnic groups, which has determined its particular genetic composition (normal, polymorphic, and deleterious alleles). We have therefore taught students the importance of such features so they can adopt an accurate genetic approach to Colombian patients. Since the North American population also reflects a particular mixing of ethnic components, we recommend that Press and Bodurtha ${ }^{1}$ include this vital competency in their milestone template. Third, our students can become involved in some of our research group's activities in which projects and results are discussed in detail. These activities have allowed medical students to propose research approaches aimed at resolving genetic questions.

We have also observed that, although knowledge of foreign languages is not a learning objective for the elective in genetics, it is a competency that medical students should acquire during their studies. Although most medical and scientific information is available in English, globalization has implied that the medical profession must be capable of dealing with patients from diverse cultural origins who communicate in multiple languages.

We thank Drs Press and Bodurtha for their suggestions for a milestone template for evaluating medical student teaching/ learning of genetics as it enriches competencies that we are constantly evaluating. We propose that it could be enriched by including other competencies and invite other professors to use it. We would like to thank Prof. Ana Gómez for critical reading of the manuscript.

\section{SUPPLEMENTARY MATERIAL}

Supplementary material is linked to the online version of the paper at http://www.nature.com/gim

\section{DISCLOSURE}

The authors declare no conflict of interest.

Paul Laissue, $M D, P h D^{1}$, Carlos M. Restrepo, $M D, P h D^{1}$ and Angela María Ortiz, $M D, M S c^{1}$

${ }^{1}$ Center For Research in Genetics and Genomics (CIGGUR). GENIUROS Research Group, School of Medicine and Health Sciences. Universidad del Rosario. Bogota, Colombia. Correspondence: Paul Laissue (paul.laissue@urosario.edu.co)

\section{REFERENCES}

1. Press KR, Bodurtha J. Milestones for medical students completing a clinical genetics elective. Genet Med 2017;19:236-239.

2. Quintero GA. Medical education and the healthcare system-why does the curriculum need to be reformed? BMC Med 2014;12:213.

3. Mitropoulos K, Al Jaibeji H, Forero DA, et al. Success stories in genomic medicine from resource-limited countries. Hum Genomics 2015;18:9-11.

Advance online publication 11 May 2017. doi:10.1038/gim.2017.62 